\title{
A single center clinical analysis of children with neuroblastoma
}

\author{
JING-BO SHAO ${ }^{1}$, ZHENG-HUA LU ${ }^{1}$, WEN-YAN HUANG ${ }^{2}$, ZHI-BAO LV $^{3}$ and HUI JIANG ${ }^{1}$ \\ Departments of ${ }^{1}$ Hematology/Oncology, ${ }^{2}$ Nephrology and ${ }^{3}$ General Surgery, Shanghai Children's Hospital, \\ Shanghai Jiao Tong University, Shanghai 200040, P.R. China
}

Received September 27, 2014; Accepted June 16, 2015

DOI: $10.3892 / 01.2015 .3588$

\begin{abstract}
In the present study, the cases of 59 children diagnosed with neuroblastoma (NB) were retrospectively analyzed to assess the association between the short-term efficacy of treatment and prognostic factors. In total, 59 patients with NB that were diagnosed between July 1, 2008 and June 30, 2013 at Shanghai Children's Hospital were enrolled in the present study. The follow-up was performed until December 31, 2013, and the data revealed that 44 patients (74.6\%) achieved complete remission (CR) or partial remission (PR). The 3-year overall survival (OS) rate of patients with stage I, II, III, IV and IVs disease was 100, 100, 65.6, 34.8 and $85.7 \%$, respectively $(\mathrm{P}=0.02)$. The 3 -year $\mathrm{OS}$ and event-free survival rates were evidently increased in patients with favorable histology compared with the rates in the patients with unfavorable histology $(\mathrm{P}=0.046$ and 0.030 , respectively). Univariate statistical analysis revealed that the factors significantly associated with prognosis were patient age, tumor stage and risk group $(\mathrm{P}=0.004,0.02$ and 0.001 , respectively). The present study identified that tumor stage, risk group and patient age are important prognostic factors for NB. An age of 18 months was also hypothesized to be the cut-off for the prognosis of patients.
\end{abstract}

\section{Introduction}

Neuroblastoma (NB) is the second most common extracranial malignant tumor that occurs in childhood and is the most common solid tumor of infancy (1). NB is a high-grade malignancy that demonstrates a poor prognosis when metastasis occurs in the early phase of disease (2). This tumor remains an enigmatic challenge for clinical and non-clinical scientists (3). Over the past several decades, the outcome for childhood cancer has improved dramatically in the USA, but

Correspondence to: Dr Hui Jiang, Department of Hematology/Oncology, Shanghai Children's Hospital, Shanghai Jiao Tong University, No. 24 Lane 1400, West Beijing Road, Shanghai 200040, P.R. China

E-mail: jhui0111@126.com

Key words: neuroblastoma, prognosis, children, efficacy the long-term outcome of children with NB that are classified as high risk remains poor, despite the administration of aggressive multimodal therapy (2,4-6). A notable gap remains in the treatment of NB between China and the USA. For example, a previous study reported that four-year event-free survival (EFS) rate of stage IV neuroblastoma was $29.2 \%$ in China following multimodal therapy (7), while an American study revealed that the five-year EFS rate of stage IV patients with metastatic disease limited to the distant lymph nodes was $77.0 \%$ (8).

In the present study, the cases of 59 children with NB that were admitted to Shanghai Children's Hospital (Shanghai Jiao Tong University, Shanghai, China) between July 2008 and June 2013 were retrospectively analyzed, and the association between short-term efficacy and prognostic factors was retrospectively analyzed in the patients with NB.

\section{Patients and methods}

Patients. In total, 61 patients were admitted to the Department of Hematology/Oncology of Shanghai Children's Hospital between July 2008 and June 2013. As 2 patients had not received 2 courses of chemotherapy, these cases were excluded, and the 59 eligible cases were included in the present study. The patients consisted of 35 males and 24 females, with a median age of 24 months (43 days-108 months). Follow-up was performed until December 31, 2013, resulting in a median follow-up time of 22 months (4-66 months). Written informed consent was obtained from all patients and the study was approved by the ethics committee of Shanghai Children's Hospital.

The initial diagnostic testing included chest computed tomography (CT) or X-ray, and abdominal CT or B-scan ultrasonography to evaluate the size of the primary tumor, and regional extent and distant spread of disease. B-scan ocular ultrasonography and fundus examination, unilateral iliac bone marrow punctures (smear and immune phenotype) and whole body bone scans were also performed. According to the International Neuroblastoma System Study (INSS), the patients with NB were divided into different stages (9). The principle of risk group with or without MYCN data is reported in Table I.

Therapy. According to the standard treatment of NB administered at the Department of Hematology/Oncology of the Shanghai Children's Hospital, the patients underwent surgical treatment first, and were then diagnosed subsequent to tumor 
resection. The requirement for a second surgical resection was based upon the assessment following 3-5 courses of chemotherapy, if complete resection was not performed at the time of diagnosis. Subsequent to achieving complete response (CR) or partial remission (PR), 4 courses of chemotherapy were administered, leading to a total administration of $<12$ chemotherapy courses.

The low risk group was followed up closely subsequent to surgery, while the middle risk and high risk groups were administered with $160 \mathrm{mg} / \mathrm{m}^{2} 13$-cis-retinoic acid for 14 days every month for 6 months subsequent to the completion of chemotherapy.

The chemotherapy regimens consisted of the administration of cyclophosphamide, anthracyclines, platinum and other antineoplastic agents. The medium risk group received $1.5 \mathrm{mg} / \mathrm{m}^{2}$ vincristine, $120.0 \mathrm{mg} / \mathrm{m}^{2}$ cyclophosphamide, $90.0 \mathrm{mg} / \mathrm{m}^{2}$ cisplatin and $160.0 \mathrm{mg} / \mathrm{m}^{2}$ etoposide, comprising the OPEC regimen, and $1.5 \mathrm{mg} / \mathrm{m}^{2}$ vincristine, $120.0 \mathrm{mg} / \mathrm{m}^{2}$ cyclophosphamide, $90.0 \mathrm{mg} / \mathrm{m}^{2}$ cisplatin and $30.0 \mathrm{mg} / \mathrm{m}^{2}$ adriamycin, comprising the OPAC regimen, for 2-10 cycles (median, 4 cycles). The high risk group received $1.5 \mathrm{mg} / \mathrm{m}^{2}$ vincristine, $1.0 \mathrm{~g} / \mathrm{m}^{2}$ cyclophosphamide, $25.0 \mathrm{mg} / \mathrm{m}^{2}$ cisplatin and $100.0 \mathrm{mg} / \mathrm{m}^{2}$ etoposide, comprising the A regimen, and $1.5 \mathrm{~g} / \mathrm{m}^{2}$ ifosfamide, $30.0 \mathrm{mg} / \mathrm{m}^{2}$ pirarubicin and $550.0 \mathrm{mg} / \mathrm{m}^{2}$ carboplatin, comprising the $\mathrm{B}$ regimen, for 2-12 cycles, with a median treatment duration of 10 cycles. The drugs and dosages administered are reported in Table II.

Histopathology. In total, 45 patients $(45 / 59 ; 76.3 \%)$ were diagnosed by tumor pathology, according to the International Neuroblastoma Pathology Classification (INPC) system (10,11). Typical imaging characteristics and the presence of NB cells in the bone marrow, or a level of urinary VMA 2 times higher than normal, measured over 24 hours, were used to diagnose 14 patients. These diagnoses were confirmed pathologically, as the patients underwent resection subsequent to chemotherapy.

Pathological categorization of the tumors with favorable histology $(\mathrm{FH})$ or unfavorable histopathology (UH) was based on a classification system according to the quantity of Schwannian stroma, degree of differentiation, mitosis karyorrhectic index (MKI) and age at diagnosis (12).

Amplification of MYCN. Amplification of the MYCN oncogene was initially identified in NB patients in 1983, and was one of the first genes demonstrated to be of prognostic value in pediatric oncology (13). MYCN amplification continues to be of use as a marker of high-risk disease for patients with locoregional NB (14). Fluorescence in situ hybridization was utilized to identify MYCN amplification, as previously described (14).

Statistical analyses. Prior to and following 2 courses of chemotherapy during ongoing treatment, and every 3-6 months subsequent to the completion of treatment, imaging examinations, which consisted of $\mathrm{CT}$, magnetic resonance imaging and B-scan ultrasonography, were performed to evaluate the efficacy of treatment. According to internationally proposed criteria for the response to therapy in patients with $\mathrm{NB}, \mathrm{CR}$ was defined as a definite absence of the tumor, while PR was defined as a reduction in tumor size of $>50 \%$ (15).

The overall survival (OS) time was calculated as the time between diagnosis and mortality, while the event-free survival (EFS) time was calculated as the time between diagnosis and relapse, progression, secondary malignancy or mortality, or until the time of the last contact with the patient if none of these events occurred. The OS and EFS were calculated using the Kaplan and Meier method, and the curves were compared using the Mantel-Cox log-rank test. All statistical analyses were performed using GraphPad Prism 5 software (GraphPad Software, Inc., La Jolla, CA, USA). P $<0.05$ was considered to indicate a statistically significant difference.

\section{Results}

Efficiency of treatment. According to the INSS criteria, 4 patients were diagnosed with stage I disease (6.8\%), 7 patients were diagnosed with stage II disease (11.9\%), 18 patients were diagnosed with stage III disease (30.5\%), 22 patients were diagnosed with stage IV disease (37.3\%), and 8 patients were diagnosed with stage IVs disease (13.5\%). Follow-up was performed until December 31, 2013. It was found that 44 patients $(44 / 59 ; 74.6 \%)$ achieved CR or PR, 8 patients $(8 / 59$; $13.6 \%)$ experienced relapse or metastasis and 7 patients $(7 / 59$; $11.8 \%)$ discontinued treatment or follow up. In total, $1(1.7 \%)$, $30(50.8 \%)$ and $28(47.5 \%)$ patients were classified as low, medium and high risk, respectively. The differences between the 3-year OS and EFS rates in the various stages and risk groups were significantly different $(\mathrm{P}<0.05$; Figs. 1 and 2$)$. The rates of $\mathrm{CR}$ and $\mathrm{PR}$ were significantly increased in patients aged $<18$ months $(92.3 \%)$ compared with the CR and PR rates in patients aged $>18$ months (60.6\%; Fig. 3 ; Table III).

Of the 59 cases, the records for 20 patients contained data on the tumor MCYN expression. MYCN amplification was detected in 9 tumor samples $(9 / 20 ; 45 \%)$, consisting of 7 specimens with UH and 2 specimens with $\mathrm{FH}$. MYCN amplification was detected in 3 bone marrow samples $(3 / 24 ; 12.5 \%)$ that were obtained from patients with stage IV disease, out of the total 24 records that included bone marrow MCYN data.

Histopathology. In total, 45 diagnostic specimens, which consisted of 36 specimens of NB tissue and 9 specimens of ganglioneuroblastoma (GNB) tissue, were used in the present study. Overall, 3 tumors were Schwannian stroma-rich tumors $(3 / 45 ; 6.7 \%)$, of which 2 were classified as intermixed GNB (GNBi) and 1 was classified as nodular GNB (GNBn), according to the INPC system. In total, 42 patients possessed Schwannian stroma-poor tumors (42/45; 93.3\%; Table IV). Out of the 36 cases of NB, 25 tumors were undifferentiated or poorly differentiated $(25 / 36 ; 69.4 \%)$ and 11 tumors were differentiated $(11 / 36 ; 30.6 \%)$. Out of the 9 cases of GNB, 6 tumors were GNBi $(6 / 9 ; 66.7 \%)$ and 3 tumors were GNBn. High MKI $(\geq 4 \%)$ was observed in $64.4 \%$ of tumors $(29 / 45)$, intermediate MKI (2-4\%) was observed in $11.1 \%$ of tumors (5/45) and low MKI $(<2 \%)$ was observed in $24.4 \%$ of tumors $(11 / 45)$ (Table IV). In the 13 patients with FH, 5 were diagnosed with NB, 6 were diagnosed with GNBi and 2 were diagnosed with GNBn, while in the 32 patients with UH, 31 were diagnosed with NB and 1 was diagnosed with GNBn. 
Table I. Principle of the risk groups, with and without MYCN data.

$\begin{array}{lll}\text { Risk } & \text { Without MYCN data } & \text { With MYCN data }\end{array}$

Low $\quad$ Stage I patients aged $\leq 18$ months

Middle Stage I patients aged $>18$ months; stage III and IV patients aged $\leq 18$ months; all stage II and IVs patients

High Stage III and IV patients aged $\geq 18$ months
Stage I patients with a MYCN level $<5$-fold higher than normal, and stage II patients aged $<12$ months with a MYCN level $<5$-fold higher than normal

Stage I patients with a MYCN level $\geq 5$-fold higher than normal, other stage II patients, all stage IVs patients, stage III and IV patients aged 12-18 months with a MYCN level $<5$-fold higher than normal, and stage III and IV patients aged $<12$ months with a MYCN level $\geq 5$-fold higer than normal

All stage III and IV patients aged $>18$ months, and stage III and IV patients aged 12-18 months with a MYCN level $\geq 5$-fold higher than normal
A

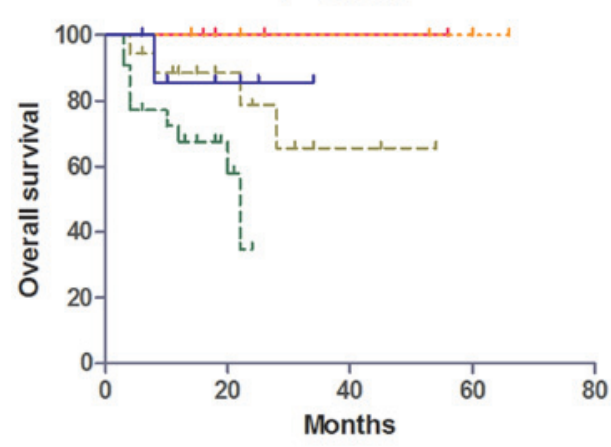

B

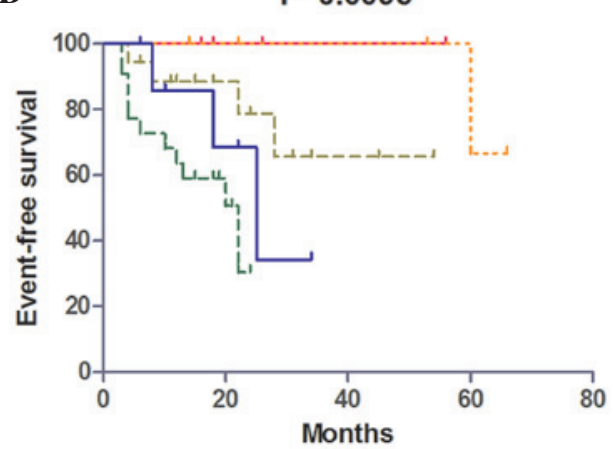

Figure 1. Kaplan-Meier (A) overall and (B) event-free survival curves in patients with neuroblastoma by tumor stage $(\mathrm{P}=0.0201$ and 0.0095$)$.

Diagnostic specimens were not obtained in 14 cases, with 13 cases being diagnosed by bone marrow metastasis and 1 case being diagnosed by clinical features, imaging and elevated VMA. In total, the diagnosis was confirmed in 12 cases by delayed surgery following 4-6 cycles of chemotherapy. Treatment was discontinued in 2 cases, without surgery, following 4 cycles of chemotherapy.

Site of the primary lesion and the relapse or metastasis site. Out of the total 59 patients, the 52 cases $(88.1 \%)$ of primary retroperitoneal tumors included 4 lesions from both the retroperitoneum and postmediastinum, 4 tumors from the postmediastinum,
Table II. Chemotherapy regimen for neuroblastoma.

\begin{tabular}{lcrcc}
\hline Regimen & Drug & $\begin{array}{r}\text { Dosage, } \\
\mathrm{mg} / \mathrm{m}^{2}\end{array}$ & $\begin{array}{c}\text { Time, } \\
\text { days }\end{array}$ & Procedure \\
\hline OPEC & VCR & 1.5 & 1 & IV \\
& CTX & 120.0 & 1 & IV gtt \\
& CDDP & 90.0 & 2 & IV gtt \\
OPAC & VP-16 & 160.0 & 4 & IV gtt \\
& VCR & 1.5 & 1 & IV \\
& CTX & 120.0 & 1 & IV gtt \\
& CDDP & 90.0 & 2 & IV gtt \\
& Adr & 30.0 & 4 & IV gtt \\
& VCR & 1.5 & 1,8 & IV \\
& CTX & 1000.0 & $1-2$ & IV gtt \\
& CDDP & 25.0 & $1-5$ & IV gtt \\
B & VP-16 & 100.0 & $1-5$ & IV gtt \\
& IFO & 1500.0 & $1-5$ & IV gtt \\
& THP & 30.0 & 1 & IV gtt \\
& CBP & 550.0 & 2 & IV gtt \\
\hline
\end{tabular}

IV, intravenous; IV gtt, intravenous guttae; VCR, vincristine; CTX, cyclophosphamide; VP-16, etoposide; CDDP, cisplatin; Adr, adriamycin; IFO, ifosfamide; THP, pirarubicin; CBP, carboplatin.

2 tumors from the neck and 1 lesion from an unknown site. The primary metastasis was located in the bone marrow in 18 patients $(18 / 59 ; 30.5 \%)$, which included 1 patient that developed metastasis in the bone marrow, bone and lung, 6 patients that developed metastasis in the bone marrow and bone, and 1 patient that developed metastasis in the bone marrow and orbit. Other incidences of metastasis included 1 case of lung and skin metastasis, 2 cases of bone metastasis, 4 cases of liver metastasis, 1 case of lung metastasis, 2 cases of skin metastasis, 1 case of distal lymph node metastasis and 1 case of liver and armpit metastasis.

In total, 8 patients, with a mean age of 41 months (range, 17 months-7 years), experienced relapse or metastasis. The time between the relapse and initial diagnosis was 4-28 months. Out of these patients, 4 experienced relapse or metastasis within 1 year of the completion of therapy, 


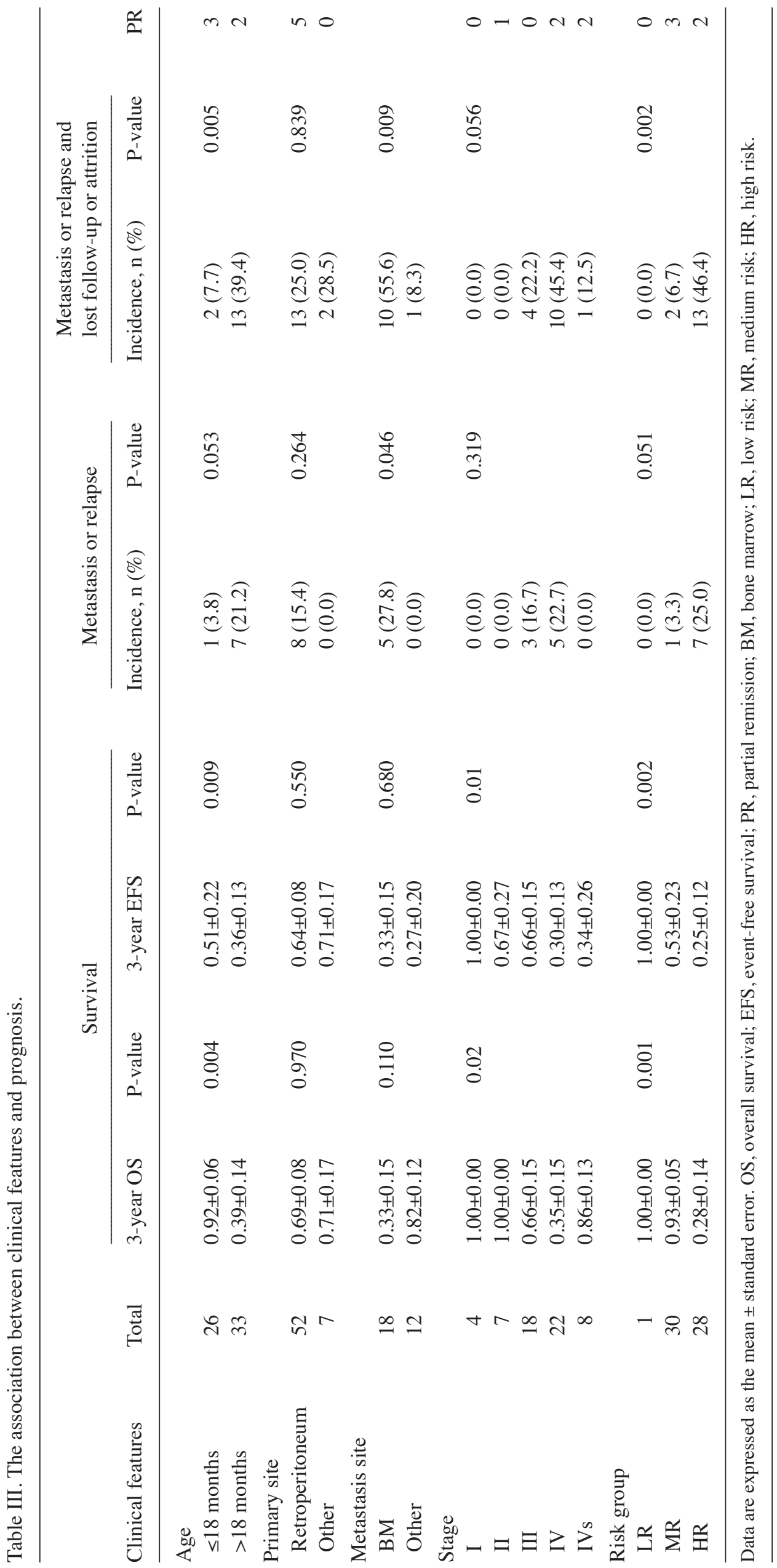


Table IV. Analysis of histological features in predicting the outcome of patients with NB.

\begin{tabular}{|c|c|c|c|c|c|}
\hline Feature & Total, $\mathrm{n}$ & 3-year OS & P-value & 3-year EFS & P-value \\
\hline \multicolumn{6}{|l|}{ Pathology } \\
\hline $\mathrm{FH}$ & 13 & $1.00 \pm 0.00$ & 0.046 & $1.00 \pm 0.00$ & 0.03 \\
\hline UH & 32 & $0.63 \pm 0.12$ & & $0.36 \pm 0.17$ & \\
\hline \multicolumn{6}{|l|}{ INPC } \\
\hline NB & 36 & $0.83 \pm 0.07$ & 0.808 & $0.45 \pm 0.19$ & 0.382 \\
\hline GNB & 9 & $0.50 \pm 0.35$ & & $0.50 \pm 0.35$ & \\
\hline \multicolumn{6}{|l|}{ Stroma } \\
\hline $\mathrm{R} / \mathrm{M}$ & 3 & $1.00 \pm 0.00$ & 0.568 & $1.00 \pm 0.00$ & 0.479 \\
\hline Poor & 42 & $0.78 \pm 0.09$ & & $0.66 \pm 0.10$ & \\
\hline \multicolumn{6}{|l|}{ MKI } \\
\hline $\mathrm{H} / \mathrm{I}$ & 34 & $0.64 \pm 0.13$ & 0.718 & $0.49 \pm 0.13$ & 0.794 \\
\hline Low & 11 & $0.59 \pm 0.19$ & & $0.59 \pm 0.19$ & \\
\hline \multicolumn{6}{|c|}{ Differentiation } \\
\hline UD/PD & 25 & $0.86 \pm 0.08$ & 0.458 & $0.44 \pm 0.19$ & 0.943 \\
\hline $\mathrm{D}$ & 11 & $0.81 \pm 0.12$ & & $0.81 \pm 0.12$ & \\
\hline
\end{tabular}

Data are expressed as the mean \pm standard error. FH, favorable histology; UH, unfavorable histology; INPC, International Neuroblastoma Pathology Classification; NB, neuroblastoma; GNB, ganglioneuroblastom; R/M, rich/mix; H/I, high/intermediate; UD/PD, undifferentiated/poorly differentiated; D, differentiated; OS, overall survival; EFS, event-free survival; SE, standard error.

Table V. Relapse and metastasis of patients with neuroblastoma.

\begin{tabular}{lcccclrr}
\hline Patient & $\begin{array}{c}\text { Age, } \\
\text { months }\end{array}$ & Stage & $\begin{array}{c}\text { Risk } \\
\text { group }\end{array}$ & 1st pathology & \multicolumn{2}{c}{ Primary site } & $\begin{array}{c}\text { Relapse } \\
\text { time, months } \\
\text { metastasis site }\end{array}$ \\
\hline 1 & 24 & III & HR & GNBn & Retroperitoneum & 28 & Intracranial \\
2 & 84 & III & HR & NB UD & Retroperitoneum & Abdominal \\
3 & 48 & III & HR & NB UD & Retroperitoneum & Abdominal \\
4 & 48 & IV & HR & BM & Retroperitoneum, BM, bone & 22 & Intracranial \\
5 & 17 & IV & MR & BM & Retroperitoneum, BM, bone & Abdominal \\
6 & 46 & IV & HR & BM & Retroperitoneum and & 22 & Intracranial \\
7 & 25 & IV & HR & BM & Retroperitoneum, BM, orbit & 20 & Intracranial \\
8 & 38 & IV & HR & BM & Retroperitoneum, BM & 4 & Lung \\
\hline
\end{tabular}

HR, high risk; MR, medium risk; GNBn, nodular ganglioneuroblastoma; NB UD, undifferentiated neuroblastoma; BM, bone marrow.

while 4 patients experienced relapse or metastasis during therapy. Overall, 3 patients developed recurrence in the retroperitoneum, 4 patients developed intracranial metastasis and 1 patient developed lung metastasis (Table V).

\section{Discussion}

Age is an important clinical prognostic factor for patients with NB. The rates of NB tumor progression and response are often age-dependent (6). The currently accepted age cut-off of 365 days was based on observations by Breslow and McCann $>30$ years ago, which indicated that an older age was associated with a worse outcome (16). An age of 12 months was hypothesized to be a boundary to determine the prognosis in NB patients, particularly for stage IV patients (17). A retrospective analysis of 3,666 patients with NB performed by the Pediatric Oncology Group and Children's Cancer Group (CCG) revealed the prognostic contribution of age to outcome was continuous in nature (2). Within the clinically relevant risk stratification, statistical support exists for an age cut-off of 460 days (18). According to a previous study by CCG, patients aged between 12 and 18 months of age demonstrate a significantly improved long-term survival rate compared with older children treated with intensive chemotherapy, with or without autologous bone marrow transplantation (19). These patients may not benefit from additional intensification of therapy beyond that provided in earlier clinical trials and may even maintain this high survival rate with less intensive therapy $(19,20)$. 
A

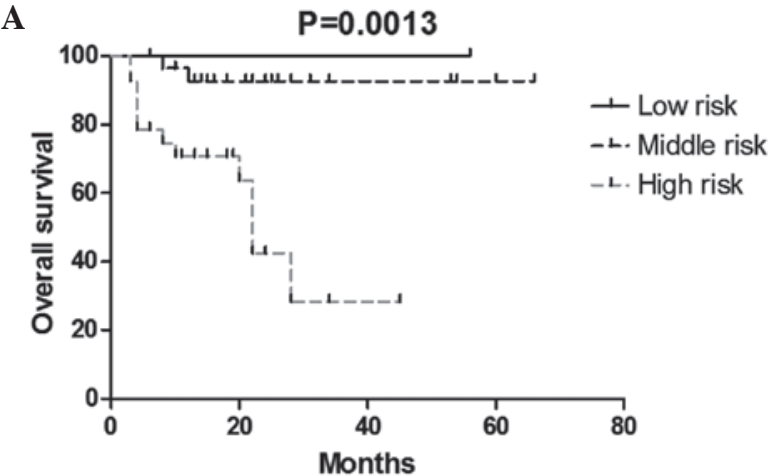

B

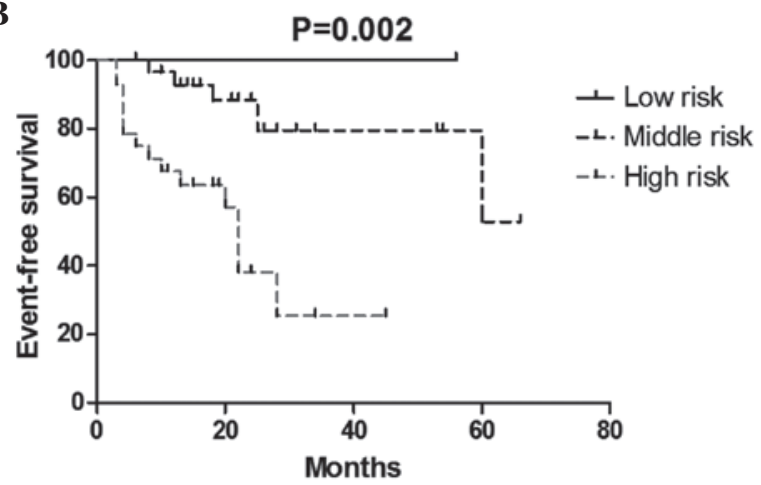

Figure 2. Kaplan-Meier (A) overall and (B) event-free survival curves of patients with neuroblastoma by risk group $(\mathrm{P}=0.0013$ and 0.002$)$.

The potential use of a younger age as a prognostic factor in patients with NB was confirmed in the present study. The 3-year OS and EFS rates in patients aged $<18$ months were significantly increased compared with patients aged $>18$ months $(\mathrm{P}=0.004$ and 0.009 , respectively), while the rates of relapse and metastasis and lost follow up/abandonment decreased significantly in patients at a younger age. The age cut-off of 18 months may be considered as a boundary, which causes patients aged between 12 and 18 months of age to be classified as a middle risk group and receive low-dose chemotherapy, avoiding the cardiotoxicity caused by anthracyclines.

A previous study by Hero et al (21) provided strong evidence that spontaneous regression may regularly be observed in localized infant NB and was not limited to the first year of life. Tumor regression without cytotoxic therapy was observed in $47 \%$ of the infant patients with non-resected NB in the study by Hero et al. A wait-and-see strategy was justified in those patients. The long-term outcome of patients with stage IVs NB revealed that the majority of patients demonstrate no clinically or radiologically significant sequelae but do exhibit residual abnormalities (22). However, not all infants with NB demonstrate a good prognosis, and neonates with hepatomegaly are particularly at risk (23).

The literature has previously reported the survival rate for symptomatic infants with stage IVs NB that were $\leq 2$ months of age was lower compared with the survival rate for infants with symptomatic NB $>2$ months of age (78 vs. $90 \%$ ), suggesting that extremely young infants with symptomatic NB are at the highest risk of succumbing to the disease (24). These infants with rapidly expanding liver disease may require an earlier administration of a more
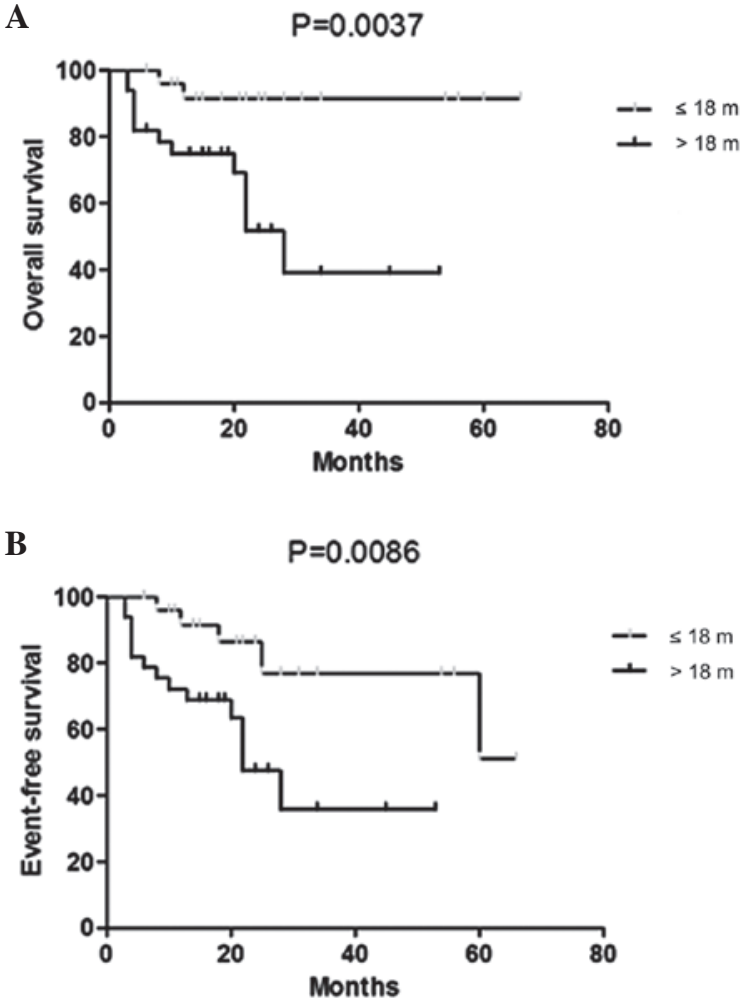

Figure 3. Kaplan-Meier (A) overall and (B) event-free survival of patients with neuroblastoma by age $(\mathrm{P}=0.0037$ and 0.0086$)$.

aggressive chemotherapy, to decrease the risk of abdominal distention, resulting in mechanical respiratory failure. The treatment administered to infants with stage IVs NB with massive hepatomegaly should be individualized based on the disease course (25). A sequential approach comprising observation, intravenous chemotherapy and hepatic intra-arterial chemoembolization may improve the outcome of these infants $(26,27)$.

In the present study, 8 patients with stage IVs NB survived without recurrence, and disease progression may be associated with the receipt of intervention immediately subsequent to diagnosis. One patient received surgery only, but follow-up was discontinued subsequent to 7 months of CR. The remaining 7 patients received chemotherapy, with 5 patients achieving CR and 2 patients achieving PR, due to the presence of minimal residual disease indicated on imaging. Minimal residual disease indicated on imaging investigation performed subsequent to the end of treatment may indicate the presence of a non-tumor tissue, or the immune system may effectively remove minimal residual tumor; however, a small number of tumor cells may continue to possess self-differentiation properties in the body environment. The incidence of minimal residual disease decreased the EFS rate, but did not affect the OS rate. No large study investigating minimal residual disease in imaging examinations has been performed, so the characteristics of non-tumor tissue and the process of tumor differentiation remain unknown. This requires investigation in a future study.

Survival subsequent to NB relapse is poor. In a retrospective study, Garaventa et al (28) reported the cases of 781 children with NB that experienced tumor recurrence. 
The 10 -year OS rate was $6.8 \%$ following progression and $14.4 \%$ following relapse. The majority of relapses occurred early (median interval, 7.8 months), but 86 (24\%) occurred late (median, 28 months). Early relapses had a more rapid, unfavorable course, with $\sim 80 \%$ of mortalities occurring within 2 years, whereas the survival time was longer for late relapses. At present, children are offered a wide variety of salvage regimens following relapse (29-31). Multiple salvage therapy has been reported in a previous study to extend the median survival time between 2.2 and 3.2 months for patients with MYCN amplification and between 0.7 and 5.8 months for patients with early relapse subsequent to stem cell transplantation, but the long-term disease-free survival following recurrent disease remains extremely poor (32). This study reported the cases of 8 patients that experienced recurrence or metastasis, with 4 patients experiencing late-occurring intracranial metastases (median, 23 months). In total, 8 patients discontinued therapy following relapse or metastasis.

$\mathrm{NB}$ is classified as a small blue round cell neoplasm of childhood and belongs to the group of peripheral neuroblastic tumors, which includes NB, GNB and the benign ganglioneuromas (33). The NB category comprises three subtypes, consisting of the undifferentiated, poorly differentiated and differentiated subtypes. These tumor types reflect different degrees of maturation, such as undifferentiated cells with large dense nuclei and scant cytoplasm, poorly differentiated and differentiating cells, or ganglion cells with the inclusion of neutrophils and Schwann cells with increased maturation (14).

High cell turnover, as manifested by increased mitotic activity at diagnosis has been recognized as a sign of aggressive tumor behavior in NB (34). Ambros et al (35), in an analysis of individual morphological features, reported that a high MKI and prominent nucleoli in undifferentiated and poorly differentiated neuroblasts at the time of diagnosis led to a poor outcome. The prognosis of the 45 patients included in this previous study was not significantly different between patients with NB and those with GNB, patients with undifferentiated or poorly differentiated NB and those with differentiated NB, patients with a high or medium MKI and those with a low MKI, and patients with stroma-rich lesions and those with stroma-poor lesions. However, the prognosis between patients with $\mathrm{FH}$ and $\mathrm{UH}$ was significantly different $(\mathrm{P}=0.046)$. In the present study, only the pathology at diagnosis was investigated, while various pathologies and the association with prognosis following chemotherapy should be assessed in additional studies.

\section{Acknowledgments}

The authors would like to thank the Shanghai Children's MedicalCenterHematology/Oncology Group, which provided numerous suggestions for the present study. This abstract was presented in part at the 46th Congress of the International Society of Pediatric Oncology, October 22-25 2014 in Toronto, ON, and was published as abstract EP-365 in Pediatr Blood Cancer 61: 2014. The present study was also supported by a grant from the Science Foundation of Shanghai Municipal Health Bureau (grant no. 201440432).

\section{References}

1. Park JR, Eggert A and Caron H: Neuroblastoma: Biology, prognosis, and treatment. Hematol Oncol Clin North Am 24: 65-86, 2010.

2. London WB, Castel V, Monclair T, Ambros PF, Pearson AD, Cohn SL, Berthold F, Nakagawara A, Ladenstein RL, Iehara T and Matthay KK: Clinical and biologic features predictive of survival after relapse of neuroblastoma: A report from the International Neuroblastoma Risk Group Project. J Clin Oncol 29: 3286-3292, 2011.

3. Castel V, Grau E, Noguera R and Martínez F: Molecular biology of neuroblastoma. Clin Transl Oncol 9: 478-483, 2007.

4. London WB, Boni L, Simon T, Berthold F, Twist C, Schmidt ML, Castleberry RP, Matthay KK, Cohn SL and De Bernardi B: The role of age in neuroblastoma risk stratification: The German, Italian, and children's oncology group perspectives. Cancer Lett 228: 257-266, 2005.

5. Pearson AD, Pinkerton CR, Lewis LJ, Imeson J, Ellershaw $\mathrm{C}$ and Machin D ; European Neuroblastoma Study Group; Children's Cancer and Leukaemia Group (CCLG formerly United Kingdom Children's Cancer Study Group): High-dose rapid and standard induction chemotherapy for patients aged over 1 year with stage 4 neuroblastoma: A randomised trial. Lancet Oncol 9: 247-256, 2008.

6. Kushner BH, Kramer K, LaQuaglia MP, Modak S and Cheung NV: Neuroblastoma in adolescents and adults: The Memorial Sloan-Kettering experience. Med Pediatr Oncol 41: 508-515, 2003.

7. Tang SQ, Huang DS, Wang JW, Feng C and Yang G: Long-term effect of high dose chemotherapy combined with stem cell transplantation on stage IV neuroblastoma in children. Zhongguo Dang Dai Er Ke Za Zhi 8: 93-96, 2006 (In Chinese).

8. Morgenstern DA, London WB, Stephens D, Volchenboum SL, Hero B, Di Cataldo A, Nakagawara A, Shimada H, Ambros PF, Matthay KK, et al: Metastatic neuroblastoma confined to distant lymph nodes (stage $4 \mathrm{~N}$ ) predicts outcome in patients with stage 4 disease: A study from the International Neuroblastoma Risk Group Database. J Clin Oncol 32: 1228-1235, 2014.

9. Brodeur GM, Pritchard J, Berthold F, Carlsen NL, Castel V, Castelberry RP, De Bernardi B, Evans AE, Favrot M and Hedborg F: Revisions of the international criteria for neuroblastoma diagnosis, staging, and response to treatment. J Clin Oncol 11: 1466-1477, 1993.

10. Umehara S, Nakagawa A, Matthay KK, Lukens JN, Seeger RC, Stram DO, Gerbing RB and Shimada H: Histopathology defines prognostic subsets of ganglioneuroblastoma, nodular. Cancer 89: 1150-1161, 2000.

11. Radner H, Blümcke I, Reifenberger G and Wiestler OD: The new WHO classification of tumors of the nervous system 2000. Pathology and genetics. Pathologe 23: 260-283, 2002 (In German).

12. Peuchmaur M: Peripheral neuroblastic tumors: Anatomo pathological classification. Ann Pathol 24: 556-567, 2004 (In French).

13. Øra I and Eggert A: Progress in treatment and risk stratification of neuroblastoma: Impact on future clinical and basic research. Semin Cancer Biol 21: 217-228, 2011.

14. Schwab M, Alitalo K, Klempnauer KH, Varmus HE, Bishop JM, Gilbert F, Brodeur G, Goldstein M and Trent J: Amplified DNA with limited homology to myc cellular oncogene is shared by human neuroblastoma cell lines and a neuroblastoma tumour. Nature 305: 245-248, 1983.

15. Monclair T, Brodeur GM, Ambros PF, Brisse HJ, Cecchetto G, Holmes K, Kaneko M, London WB, Matthay KK, Nuchtern JG, et al; INRG Task Force: The International Neuroblastoma Risk Group (INRG) staging system: An INRG Task Force report. J Clin Oncol 27: 298-303, 2009.

16. Breslow N and McCann B: Statistical estimation of prognosis for children with neuroblastoma. Cancer Res 31: 2098-2103, 1971.

17. Sung KW, Lee SH, Yoo KH, Jung HL, Cho EJ, Koo HH, Lee SK, Kim J, Lim DH, Suh YL and Kim DW: Tandem high-dose chemotherapy and autologous stem cell rescue in patients over 1 year of age with stage 4 neuroblastoma. Bone Marrow Transplant 40: 37-45, 2007.

18. London WB, Castleberry RP, Matthay KK, Look AT, Seeger RC, Shimada H, Thorner P, Brodeur G, Maris JM, Reynolds CP and Cohn SL: Evidence for an age cutoff greater than 365 days for neuroblastoma risk group stratification in the Children's Oncology Group. J Clin Oncol 23: 6459-6465, 2005. 
19. Schmidt ML, Lal A, Seeger RC, Maris JM, Shimada H, O'Leary M, Gerbing RB and Matthay KK: Favorable prognosis for patients 12 to 18 months of age with stage 4 nonamplified MYCN neuroblastoma: A Children's Cancer Group Study. J Clin Oncol 23: 6474-6480, 2005.

20. George RE, London WB, Cohn SL, Maris JM, Kretschmar C, Diller L, Brodeur GM, Castleberry RP and Look AT: Hyperdiploidy plus nonamplified MYCN confers a favorable prognosis in children 12 to 18 months old with disseminated neuroblamoma: A Pediatric Oncology Group study. J Clin Oncol 23: 6466-6473, 2005.

21. Hero B, Simon T, Spitz R,Ernestus K, Gnekow AK, Scheel-Walter HG, Schwabe D, Schilling FH, Benz-Bohm G and Berthold F: Localized infant neuroblastomas often show spontaneous regression: Results of the prospective trials NB95-S and NB97. J Clin Oncol 26: 1504-1510, 2008.

22. Levitt GA, Platt KA, De Byrne R, Sebire N and Owens CM: 4S neuroblastoma: The long-term outcome. Pediatr Blood Cancer 43: 120-125, 2004

23. Hsu LL, Evans AE and D'Angio GJ: Hepatomegaly in neuroblastoma stage $4 \mathrm{~s}$ : Criteria for treatment of the vulnerable neonate. Med Pediatr Oncol 27: 521-528, 1996.

24. Nickerson HJ, Matthay KK, Seeger RC, Brodeur GM, Shimada H, Perez C, Atkinson JB, Selch M, Gerbing RB, Stram DO and Lukens $\mathrm{J}$ : Favorable biology and outcome of stage IV-S neuroblastoma with supportive care or minimal therapy: A Children's Cancer Group Study. J Clin Oncol 18: 477-486, 2000.

25. Haupt R, Garaventa A, Gambini C, Parodi S, Cangemi G, Casale F, Viscardi E, Bianchi M,Prete A, Jenkner A, et al: Improved survival of children with neuroblastoma between 1979 and 2005: A report of the Italian Neuroblastoma Registry. J Clin Oncol 28: 2331-2338, 2010.

26. Weintraub M, Waldman E, Koplewitz B, Bloom AI, Gross E, Freeman AI and Revel-Vilk S: A sequential treatment algorithm for infants with stage $4 \mathrm{~s}$ neuroblastoma and massive hepatomegaly. Pediatr Blood Cancer 59: 182-184, 2012.

27. Weintraub M, Bloom AI, Gross E, Revel-Vilk S. Shahroor S, Koplewitz BZ and Freeman AI: Successful treatment of progressive stage 4 s hepatic neuroblastoma in a neonate with intra-arterial chemoembolization. Pediatr Blood Cancer 43: 148-151, 2004.
28. Garaventa A, Parodi S, De Bernardi B, Dau D, Manzitti C, Conte M, Casale F, Viscardi E, Bianchi M, D'Angelo P, et al: Outcome of children with neuroblastoma after progression or relapse. A retrospective study of the Italian neuroblastoma registry. Eur J Cancer 45: 2835-2842, 2009.

29. Lau L, Tai D, Weitzman S, Grant R, Baruchel S and Malkin D: Factors influencing survival in children with recurrent neuroblastoma. J Pediatr Hematol Oncol 26: 227-232, 2004.

30. London WB, Frantz CN, Campbell LA, Seeger RC, Brumback BA, Cohn SL, Matthay KK, Castleberry RP and Diller L: Phase II randomized comparison of topotecan plus cyclophosphamide versus topotecan alone in children with recurrent or refractory neuroblastoma: A Children's Oncology Group study. J Clin Oncol 28: 3808-3815, 2010.

31. Ashraf K, Shaikh F, Gibson P, Baruchel S and Irwin MS: Treatment with topotecan plus cyclophosphamide in children with first relapse of neuroblastoma. Pediatr Blood Cancer 60: 1636-1641, 2013

32. Maris JM: Recent advances in neuroblastoma. N Engl J Med 362: 2202-2211, 2010

33. Sałacińska-Łoś E, Kobos J and Taran K: Estimation of diagnostic value of chosen markers of neural differentiation in neuroblastoma group of tumors and pPNETs. Pol J Pathol 59: 195-199, 2008

34. George RE, Variend S, Cullinane C, Cotterill SJ, McGuckin AG, Ellershaw C, Lunec J and Pearson AD; United Kingdom Children Cancer Study Group: Relationship between histopathological features, MYCN amplification, and prognosis: A UKCCSG study. United Kingdom Children Cancer Study Group. Med Pediatr Oncol 36: 169-176, 2001.

35. Ambros IM, Hata J, Joshi VV, Roald B, Dehner LP, Tüchler H, Pötschger U and Shimada H: Morphologic features of neuroblastoma (Schwannian stroma-poor tumors) in clinically favorable and unfavorable groups. Cancer 94: 1574-1583, 2002. 